\title{
Anatomía de los órganos subterráneos de cuatro Dicotiledóneas medicinales rioplatenses (Argentina). II. Plantas palustres
}

\author{
Anatomy of the Underground Organs of the Four Dicotyledons \\ Medicinal Plants on the Río de la Plata Shore (Argentina). \\ II. Marsh Plants
}

\author{
Perrotta, Vanesa G.; Marcelo P. Hernández; Ana M. Arambarri* \\ Laboratorio de Morfología Comparada de Espermatófitas (LAMCE), Facultad de Ciencias Agrarias y Foresta- \\ les, Universidad Nacional de La Plata, 60 y 119, C.C. 31, (1900) La Plata, Buenos Aires, Argentina. \\ *Autor corresponsal: anaramba@yahoo.com.ar; botgral@agro.unlp.edu.ar
}

- Resumen - Perrotta, Vanesa G.; Marcelo P. Hernández; Ana M. Arambarri. 2017. "Anatomía de los órganos subterráneos de cuatro Dicotiledóneas medicinales rioplatenses (Argentina). II. Plantas palustres”. Lilloa 54 (2). Este artículo presenta los resultados del estudio morfo-anatómico e histoquímico de los órganos subterráneos de cuatro especies de Dicotiledóneas palustres usados en la medicina popular rioplatense. Las especies estudiadas son: Alternanthera philoxeroides, Eryngium pandanifolium, Ludwigia peploides y Senecio bonariensis. El objetivo de este estudio fue caracterizar las raíces y rizomas por sus atributos histológico-estructurales e identificar compuestos químicos que faciliten la diferenciación de los taxones a partir de muestras enteras o fragmentadas. Se analizó material fresco recolectado en el área de estudio, se incorporaron ejemplares al herbario (LPAG), Facultad de Ciencias Agrarias y Forestales, Universidad Nacional de La Plata. Material de rizomas y raíces fue fijado en líquido conservador. Se realizaron cortes y preparaciones histológicas, las que fueron estudiadas con microscopio óptico. Mediante técnicas histoquímicas se identificaron almidón, mucílagos, sustancias lipofílicas y taninos. Los caracteres diagnósticos estructurales fueron: el tipo de parénquima cortical; desarrollo secundario de la estructura vascular; presencia de tejidos secretores y tipos de cristales. Se generó una tabla comparativa de los compuestos histoquímicos hallados y una clave dicotómica con los caracteres de rizomas y raíces, datos que constituyen una herramienta de valor en el control de calidad botánica.

Palabras clave: Anatomía, histoquímica, Dicotiledóneas, raíces, rizomas.

Abstract - Perrotta, Vanesa G.; Marcelo P. Hernández; Ana M. Arambarri. 2017. "Anatomy of the underground organs of the four Dicotyledons medicinal plants on the Rio de la Plata shore (Argentina). II. Marsh plants". Lilloa 54 (2). The paper presents morphology-anatomy and histochemical data of the underground organs of four marshy species of Dicotyledons used in the Rio de la Plata folk medicine. They are: Alternanthera philoxeroides, Eryngium pandanifolium, Ludwigia peploides y Senecio bonariensis. The aim of this study was to obtain structural characteristics of the rhizome and roots, and identified chemical compounds that allow differentiation of taxa, facilitating the identification of whole or fragmented samples of organs used in folk medicine. Fresh material was collected in the study area. Specimens were prepared and included in the LPAG herbarium, Facultad de Ciencias Agrarias y Forestales, Universidad Nacional de La Plata. Rhizomes and roots samples were fixed in conservation liquid. Slides were made and analyzed with a light microscope. Histochemical analysis to recognize starch, mucilage, lipophilic substances, and tannins were performed. The diagnoses structural features were: cortical parenchyma type; secondary vascular tissue development; presence of secretory tissue, and crystal types. A comparative table with histochemical results and a dichotomous key using micrographic rhizome and root characters were generated to get a valuable tool to establish authenticity of botany quality control.

Keywords: Anatomy, histochemistry, Dicotyledons, rhizomes, roots. 


\section{INTRODUCCIÓN}

Este trabajo es una nueva contribución al estudio de la anatomía comparada de los órganos vegetativos de especies rioplatenses utilizados en la medicina popular. La región rioplatense se extiende desde Diamante en la provincia de Entre Ríos (32 $4^{\prime} 0^{\prime \prime}$ S $60^{\circ}$ 39'0"O) hasta Punta Lara en la provincia de Buenos Aires (3447'28"S 57059'49”O). Nuestro estudio se centra en las especies usadas en medicina popular que habitan la parte inferior del Delta del Paraná, isla Martín García y ribera del Río de la Plata, basándonos en los trabajos de Pochettino et al. (1997); Lahitte y Hurrell (1996); Lahitte et al. (2004); Hernández et al. (2009, 2010, 2013, 2015a); Hernández y Arambarri (2011). Las investigaciones anteriores, dieron origen a las siguientes publicaciones: los tallos aéreos y subterráneos de siete especies herbáceas de Monocotiledóneas (Novoa et al., 2012), la anatomía de los tallos aéreos de 37 especies de Dicotiledóneas (Arambarri et al., 2013), las raíces de 22 especies (Colares et al., 2014), las hojas de 62 especies (Hernández et al., 2015b), la anatomía foliar de Commelinaceae (Novoa y Arambarri, 2016) y la anatomía de órganos subterráneos de Monocotiledóneas medicinales, parte I (acuáticas y palustres) (Arambarri et al., 2016) que precedió a esta parte II sobre Dicotiledóneas palustres de la misma región. En los mismos, citamos la abundante bibliografía que existe sobre los humedales rioplatenses (e. g., Pasarelli et al., 2014). Las propiedades medicinales de las plantas que crecen en la región también están ampliamente estudiadas (Hieronymus, 1882; Domínguez, 1928; Toursarkissian, 1980; Lahitte y Hurrell, 1996; Lahitte et al., 2004; Alonso y Desmarchelier, 2005; Barboza et al., 2006). Algunos autores se han ocupado de profundizar sobre la actividad específica de las especies como analgésica y antifúngica (Rondina et al., 2008, 2010), en otros, se cita la actividad biológica de las especies (Alonso y Desmarchelier, 2005; Barboza et al., 2009). Existen algunas publicaciones sobre características anatómicas de plantas hidrófilas, ya sea vástagos (e.g. González, 2002) o rizomas y raíces (e.g., Schussler y Longstreth, 2000; Seago Jr. et al., 2005). Estas y otras obras citadas en la discusión, han servido como base para nuestro estudio, desarrollado con la convicción de que la profundización de los conocimientos de anatomía comparada de los órganos utilizados como drogas medicinales es de un gran valor en el proceso de control botánico.

En esta entrega, como parte del proyecto de estudio de los órganos usados en medicina popular de plantas acuáticas, palustres, trepadoras y volubles, analizamos los órganos subterráneos de cuatro Dicotiledóneas palustres: el rizoma y raíces de Senecio bonariensis y las raíces de Alternanthera philoxeroides, Eryngium pandanifolium y los dos tipos de raíces (raíz propiamente dicha y neumatóforo) formados en el estolón de Ludwigia peploides, con el propósito de obtener caracteres micrográficos e histoquímicos de los mismos, que permitan generar una clave dicotómica que constituya un patrón de identificación aplicable al control de calidad botánica.

\section{MATERIALES Y MÉTODOS}

\section{MATERIAL ESTUdiAdo}

Se recolectaron plantas en la ribera del Río de la Plata y localidades próximas, algunas muestras fueron tomadas de plantas cultivadas en el Jardín Botánico y Arboretum «C. Spegazzini» de la Facultad de Ciencias Agrarias y Forestales (JByA, FCA y F), Universidad Nacional de La Plata. Los ejemplares se incorporaron al herbario (LPAG) de la misma Institución.

Material estudiado: ARGENTINA, provincia de Buenos Aires.

Alternanthera philoxeroides (Mart.) Griseb.: Pdo. La Plata, La Plata, 14-X-2011, Arambarri 361 (LPAG); Ciudad Autónoma, V-2012, Auguet s. n. (LPAG).

Eryngium pandanifolium Cham. \& Schltdl.: Pdo. Magdalena, camino a Julio Arditi, 6-X-2015, Hernández 166 (LPAG); Reserva Natural «El Destino», Fundación Elsa Shaw de Pearson, 12-V-2016, Martínez Alonso 116 
(LPAG). Ludwigia peploides (Kunth) P.H. Raven: Pdo. Coronel Brandsen, Samborombón, 29-I-1990, Bayón s. n. (LPAG 4596); Pdo. Berisso, La Balandra, 3-III-2017, Hernández 234, 235 (LPAG).

Senecio bonariensis Hook. \& Arn.: Pdo. La Plata, City Bell, 28-II-1998, Bayón 389(d) (LPAG); Manuel B. Gonnet, 31-X-2015, Hernández162a, $b$ (LPAG).

\section{MÉTODOS}

Se analizaron las raíces de las cuatro especies estudiadas y los rizomas de Senecio bonariensis, órganos usados en la medicina popular. Se describen e ilustran los órganos analizados. En la Tabla 1 se muestran los resultados de las reacciones histoquímicas y los cristales existentes en cada órgano y especie. Los caracteres estructurales permitieron generar una clave dicotómica de las especies estudiadas. En el Apéndice 1, se presentan las especies - por orden alfabético del género- con su familia, un nombre vernáculo, usos y la actividad biológica de los órganos subterráneos.

Microscopía óptica.- Para el estudio de la anatomía, el material fresco se lavó y conservó en solución formalina-aceto-alcohólica (FAA 70\%) (Johansen, 1940). Se hicieron cortes transversales a mano alzada los que fueron seleccionados, decolorados y lavados con agua destilada tres veces y se practicaron tinciones con safranina alcohólica $80 \%$, doble coloración con azul de Alcian y safranina (Luque et al., 1996). Otra parte se deshidrató según la serie ascendente al- cohol etílico absoluto-xileno. Se infiltró con parafina, se realizaron cortes entre 10 y 15 $\mu \mathrm{m}$ de espesor con micrótomo rotativo Leitz. Para la tinción de los cortes con micrótomo se utilizó violeta de cresilo 1\% (D’Ambrogio de Argüeso, 1986). En todos los casos se montaron en gelatina-glicerina y sellaron con esmalte de uñas. Las estructuras fueron estudiadas con un microscopio óptico Leitz SM Lux. Las figuras fueron preparadas con fotografías registradas en un microscopio óptico Gemalux equipado con cámara Motic 1000 y software Motic Image Plus 2.0. La terminología está de acuerdo con Metcalfe y Chalk (1979) y la nomenclatura botánica se actualizó haciendo uso de los sitios www. darwin.edu.ar y trópicos.org (consultados: junio 2017).

Técnicas histoquímicas.- Sobre cortes del material fresco se practicaron las técnicas de identificación de almidón con Lugol (iodo y ioduro de potasio, IKI) (Ruzin, 1999); para sustancias lipofílicas se empleó una solución alcohólica saturada de Sudan IV (Zarlavsky, 2014) y Oil red «O» (Gurr, 1971); para mucílagos se utilizó azul brillante de cresilo 1\% (Cosa et al., 2014) y para sustancias fenólicas (taninos) se empleó el reactivo cloruro férrico al $10 \%$ y carbonato de sodio al $2 \%$ (Zarlavsky, 2014).

\section{RESULTADOS}

Alternanthera philoxeroides.- La estructura primaria de la raíz es triarca (Fig. 1A). La estructura secundaria anómala consiste en círculos concéntricos o disposición en es-

Tabla 1. Compuestos químicos y cristales hallados en las raíces y rizomas de las especies estudiadas. Ref.: [+] presencia; [-] ausencia.

\begin{tabular}{|c|c|c|c|c|c|}
\hline Cuencas & Almidón & Lípidos & Mucílagos & Taninos & Cristales \\
\hline Alternanthera philoxeroides & - & + & - & - & drusas \\
\hline Eryngium pandanifolium & - & ++ & - & - & drusas \\
\hline Ludwigia peploides & + & + & - & + & rafidios \\
\hline $\begin{array}{l}\text { Senecio bonariensis } \\
\text { (rizoma) }\end{array}$ & $\begin{array}{c}+ \\
\text { inulina }\end{array}$ & + & + & - & - \\
\hline $\begin{array}{l}\text { Senecio bonariensis } \\
\text { (raíz) }\end{array}$ & $\begin{array}{c}+ \\
\text { inulina }\end{array}$ & + & - & - & - \\
\hline
\end{tabular}



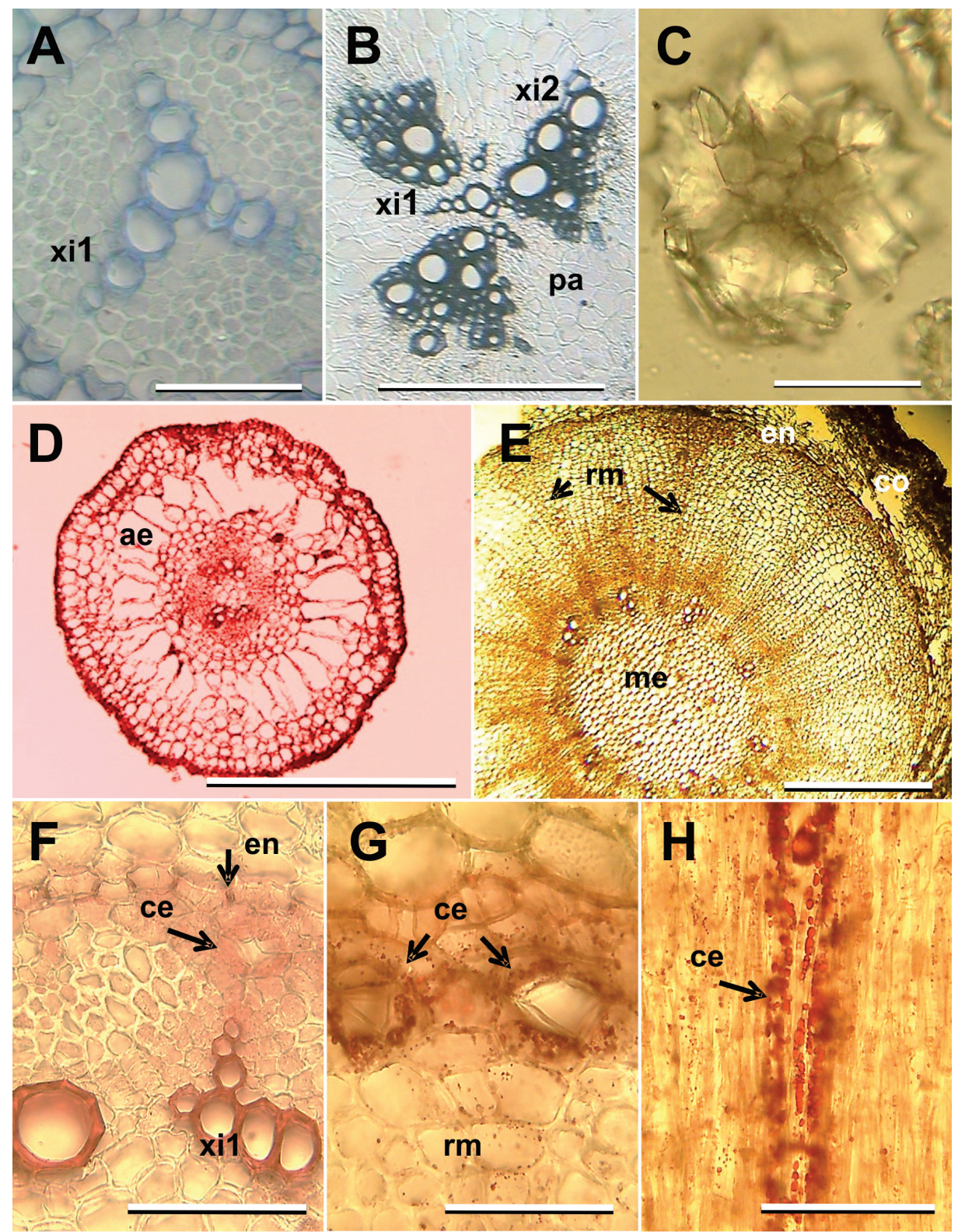

Fig. 1. Raíces, anatomía e histoquímica. A-C] Alternanthera philoxeroides: A] Cilindro central de la raíz triarca. B) Detalle de la estructura secundaria con tres cordones de tejido vascular secundario alternos con los tres cordones de xilema primario. C) Muestra de una drusa presente en el parénquima cortical. D-H) Eryngium pandanifolium: D) Estructura primaria diarca-tetrarca, con parénquima aerífero en la corteza. E) Estructura secundaria mostrando en el centro la médula, en cuya periferia se observan los vasos del xilema secundario que alternan con los cordones de xilema primario frente a los que desarrollan los radios medulares pluriseriados que hacia la periferia se van ensanchando y dilatan entre el floema secundario. F) Detalle de un conducto esquizógeno frente al cordón de xilema primario. G) Detalle de dos conductos esquizógenos en el radio medular dilatado de la estructura secundaria. H) Detalle del corte longitudinal de un conducto esquizógeno conteniendo gotas de aceites esenciales. Ref.: ae, aerénquima; ce, conducto esquizógeno; co, corteza; dr, drusa; en, endodermis; me, médula; pa, parénquima; rm, radio medular; xi1, xilema primario; xi2, xilema secundario. Escalas: A: $200 \mu \mathrm{m}$; B: $300 \mu \mathrm{m}$; C: $50 \mu \mathrm{m}$; D, E: $500 \mu \mathrm{m}$; F-H: $100 \mu \mathrm{m}$. 
piral de cordones vasculares que se originan de cámbiumes formados sucesivamente en el parénquima y conectados entre sí. En la parte central de la misma, se forman tres cordones vasculares secundarios que alternan con los tres cordones de xilema primario (Fig. 1B). En la corteza parenquimática se identificaron drusas (Fig. 1C).

Eryngium pandanifolium.- La estructura primaria muestra la rizodermis y la exodermis unistratas (Fig. 1D). La corteza es aerenquimática formando amplias cámaras radiadas, separadas por tabiques uniseriados de células redondeadas, que se extienden desde 2-3 capas de parénquima denso periférico hasta 2-3 capas de parénquima denso junto a la endodermis (Fig. 1D). La endodermis presenta banda de Caspary visible como puntos de Caspary (Fig. 1F). El cilindro central presenta el periciclo y los tejidos de conducción alternos y radiados (Fig. 1D) y médula parenquimática. En la estructura primaria, frente a cada cordón de xilema primario se visualiza un conducto secretor esquizógeno con aceites esenciales (Fig. 1F). En la estructura secundaria (Fig. 1E), la rizodermis y corteza primaria periférica son reemplazadas por la peridermis; el aerénquima es aplastado por el crecimiento secundario vascular. La endodermis se adapta al aumento de diámetro de la raíz y rodea al amplio cilindro central secundario (Fig. $1 \mathrm{E})$. En este, rodeando la amplia médula de parénquima con drusas, se hallan grupos de xilema secundario que alternan con los cordones de xilema primario. Frente a cada cordón de xilema primario se forma un radio medular pluriseriado y dilatado hacia la periferia, llegando hasta la endodermis. Entre los radios medulares se ubica el floema secundario (Fig. 1E). En el sector dilatado de los radios medulares se pueden observar uno o dos conductos esquizógenos con aceites esenciales (Fig. 1G, H).

Ludwigia peploides.- - La raíz propiamente dicha con estructura primaria (Fig. 2A) presenta desde la periferia hacia el centro: rizodermis unistrata de células pequeñas con paredes delgadas; una capa de exodermis formada por células proporcionalmente grandes, hexagonales, radialmente alargadas, con paredes suberificadas; parénquima denso de células isodiamétricas con paredes delgadas y disposición ordenada, que dejan pequeños espacios intercelulares, el cual se extiende hasta la capa más interna de la corteza, la endodermis, con el primer engrosamiento de sus paredes en banda de Caspary. En el cilindro central el periciclo presenta células rectangulares, periclinalmente alargadas, rodea los tejidos vasculares que forman una actinostela pentarca. La estructura secundaria (Fig. 2B), desarrolla peridermis donde se destaca el súber formado por 5-8 estratos sobre el que se visualiza el desprendimiento de las células de parénquima cortical, exodermis y rizodermis de la estructura primaria (Fig. 2B, C). Interno al súber se hallan 6-8 estratos de células de parénquima que rodean un estrecho anillo de floema secundario que enmarca un extenso xilema secundario caracterizado por vasos de gran tamaño, que ocupa el centro de la raíz (Fig. 2B). En el parénquima cortical y el floema abundan idioblastos con fascículos de rafidios (Fig. 2C). En el parénquima cortical, también se identificaron taninos y almidón, este polisacárido también se halló en las fibras y parénquima xilemático (Fig. 2D). La suberina fue identificada en las paredes de la endodermis, exodermis y súber. El neumatóforo es una forma de raíz esponjosa que flota en el agua, es de color blanco con un hilo oscuro en la parte central que corresponde al cilindro central. Su estructura muestra desde la periferia hacia el centro: la rizodermis y un extenso aerénquima con grandes cámaras delimitadas por células alargadas que forman círculos concéntricos (Fig. 2E, F) alrededor del cilindro central. En este, se destaca el xilema (Fig. 2E, G). Las reacciones histoquímicas dieron resultado negativo para almidón, sustancias lipofílicas, mucílagos y taninos.

Senecio bonariensis. - El rizoma de unos $5 \mathrm{~cm}$ de diámetro es fistuloso con entrenudos poco notables de $0,8-2,0 \mathrm{~cm}$ de longitud y 

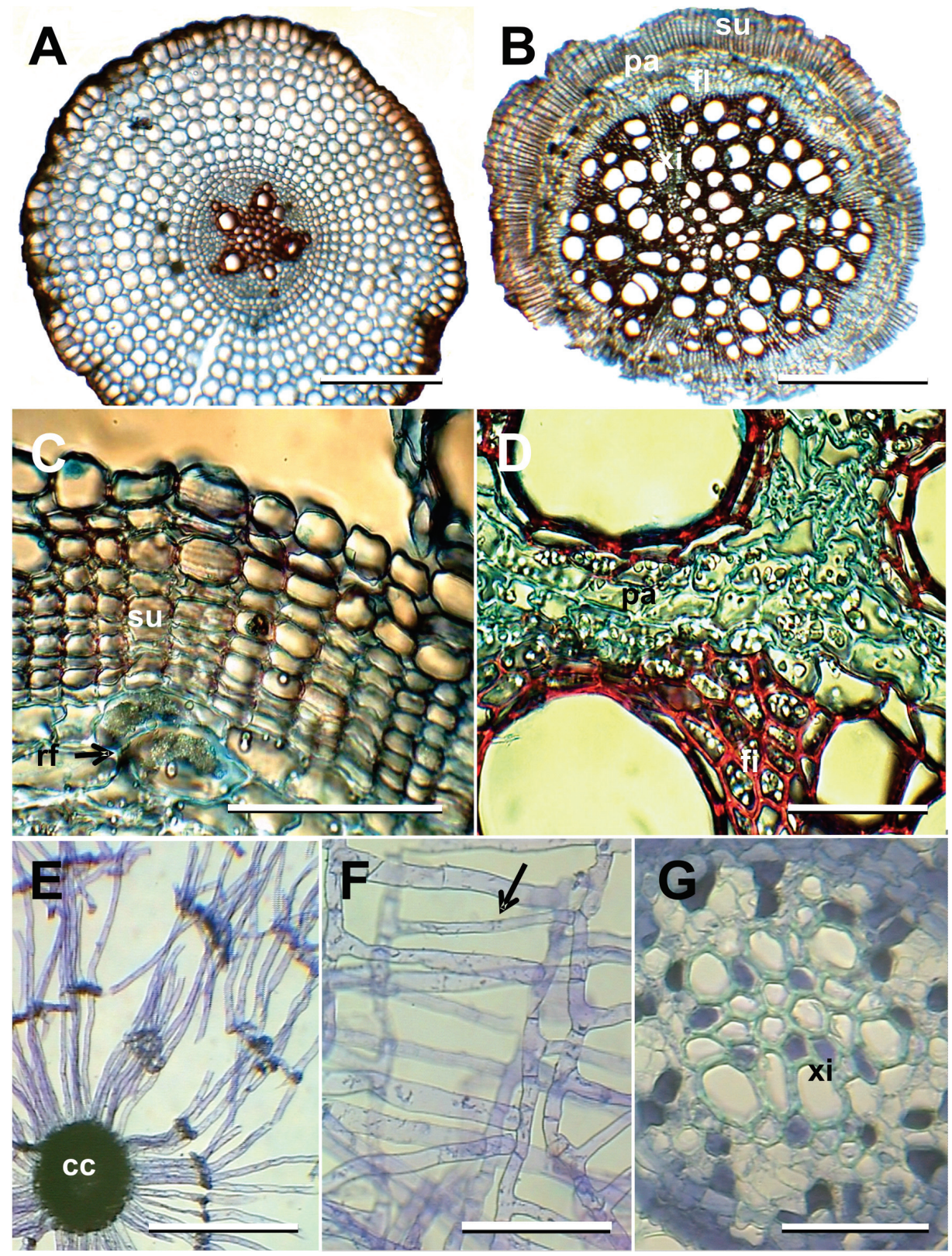

Fig. 2. Raíz y neumatóforo de Ludwigia peploides. A-DJ Raíz propiamente dicha: A] Estructura primaria. Se destacan la rizodermis unistrata, la exodermis con células proporcionalmente grandes y el parénquima cortical de células redondeadas ordenadas y separadas por pequeños espacios intercelulares. B) Estructura secundaria mostrando en la periferia el súber pluristrato, un anillo estrecho de parénquima y floema secundario y el xilema secundario que ocupa todo el centro de la raíz. C) Detalle de la ritidomis, con el súber y externamente el desprendimiento de parénquima, exodermis y rizodermis. En las células de parénquima internas al súber se detallan dos idioblastos con rafidios. D) Xilema secundario donde se destaca la presencia de almidón en el parénquima y en las fibras del xilema secundario. Neumatóforo: E) vista general del corte transversal de un neumatóforo con extenso aerénquima y pequeño cilindro central. F) Detalle de las células del aerénquima. G) Detalle del cilindro central donde se destaca el xilema. Ref.: cc, cilindro central; fi, fibras; fl, floema; pa, parénquima; rf, rafidios; su, súber; xi, xilema. Escalas: A, F, G: $200 \mu \mathrm{m}$; B, E: $500 \mu \mathrm{m}$; C, D: $50 \mu \mathrm{m}$. 
de color castaño oscuro. Presenta epidermis unistrata con pared externa lipofílica (Fig. $3 \mathrm{~A}$ ), corteza formada por parénquima aerífero de células isodiamétricas (Fig. 3A). En rizomas adultos se forman amplias cavidades lisígenas rodeadas por células de parénquima radiado (Fig. 3B). El límite de la fístula lo forman varias capas de células con paredes suberificadas (Fig. 3C). En el parénquima se identificó inulina reserva propia de las Asteraceae (Fig. 3D). La raíz con estructura secundaria inicial presenta desde la periferia hacia el centro: rizodermis y exodermis, ambas unistratas, la pared periclinal externa de la rizodermis y la totalidad de las paredes de las células de la exodermis dieron reacción positiva para sustancias lipofílicas (ceras); la corteza es amplia, formada por parénqui- ma de células redondeadas que dejan en la región periférica cámaras aerenquimáticas y hacia el centro el tamaño de las cavidades de aerénquima se reduce formando meatos y en las capas más internas, adyacentes a la endodermis, solo hay pequeños espacios intercelulares (Fig. 4A). La endodermis está formada por células periclinalmente alargadas con engrosamiento en banda de Caspary (Fig. 4B). El cilindro central presenta un notable periciclo de células periclinalmente alargadas que limita al anillo discontinuo de floema, el cual circunda al xilema secundario y este la médula reducida (Fig. 4A, B). En un estado más avanzado de la estructura secundaria el xilema ocupa el centro de la raíz y se observan los radios leñosos pluriseriados y dilatados a nivel del floema secundario

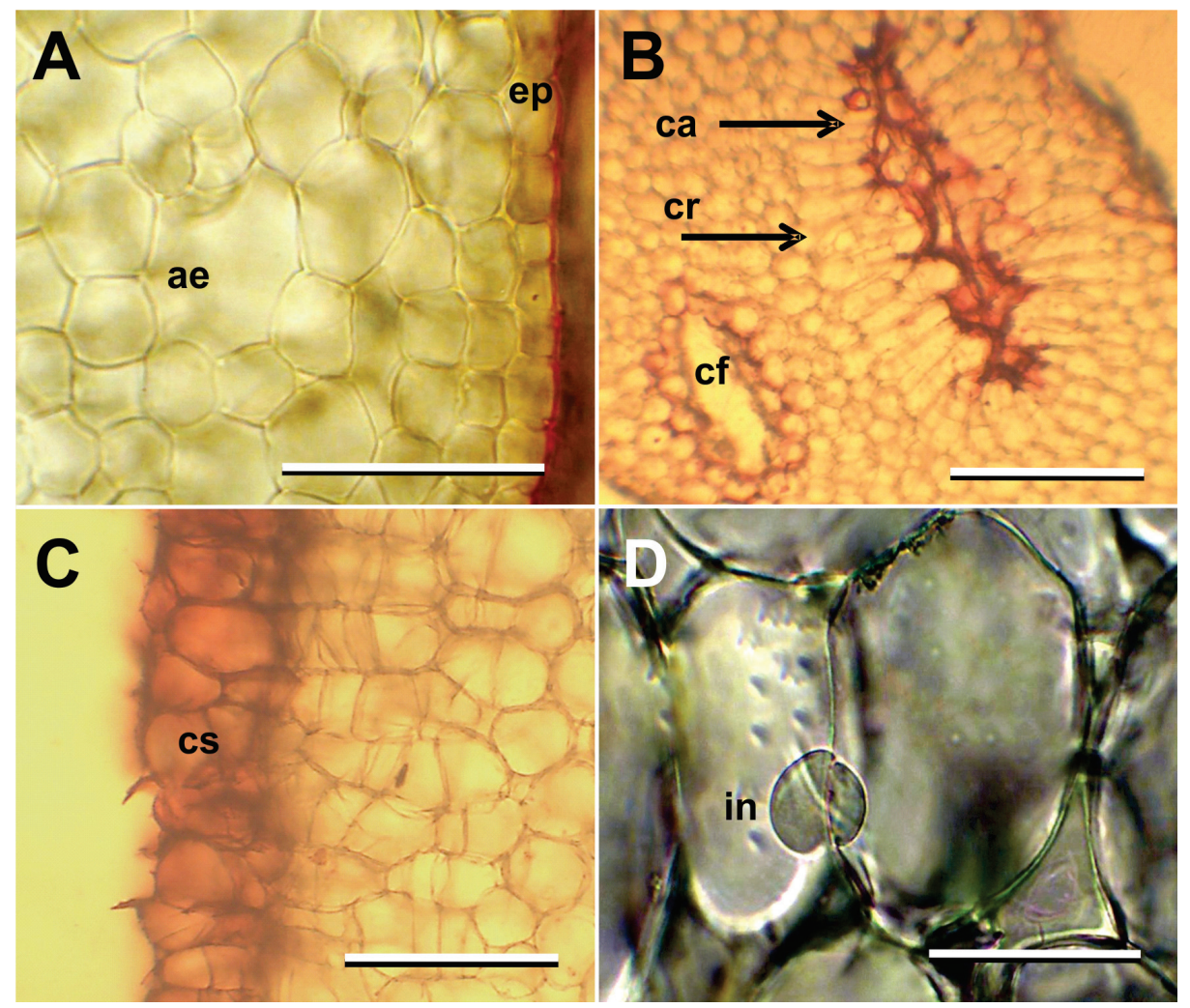

Fig. 3. Rizoma de Senecio bonariensis. A) Epidermis con cutícula, corteza aerenquimática. B) Formación de cámaras en el parénquima y una cámara desarrollada con células radiadas que la limitan. C) Células suberificadas que limitan la fístula del rizoma. D) Cristal de inulina en el parénquima. Ref.: ae, aerénquima; ca, cámara; cf, cámara en formación; cr, células radiadas; cs, células suberificadas; in, inulina. Escalas: A: $100 \mu \mathrm{m}$; B: $500 \mu \mathrm{m}$; C: $200 \mu \mathrm{m}$; D: $50 \mu \mathrm{m}$. 

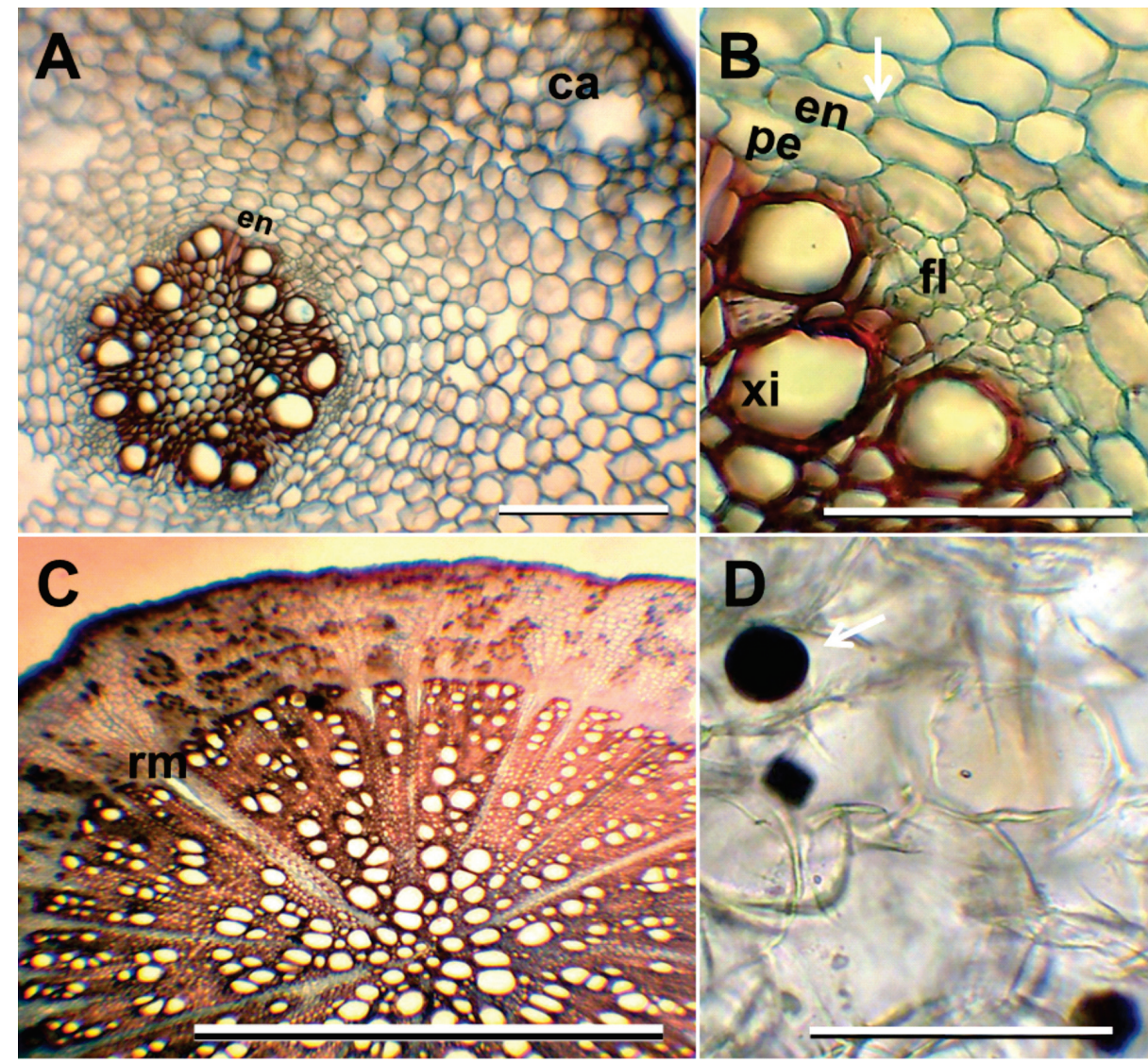

Fig. 4. Raíz de Senecio bonariensis. A) Estructura secundaria inicial con corteza primaria aerenquimática; en el cilindro central se visualiza el floema y xilema secundarios y una reducida médula parenquimática. B) Detalle de la endodermis donde se observan los puntos de Caspary; el periciclo con células proporcionalmente grandes, floema y xilema secundarios. C] Estructura secundaria en una raíz desarrollada. D) Parénquima con almidón.

Ref.: ca, cámara; en, endodermis; fl, floema secundario; pe, periciclo; rm, radio medular; xi, xilema secundario. Escalas: A: $200 \mu \mathrm{m}$; B, D: 100; C: $1000 \mu \mathrm{m}$.

(Fig. 4C). Aunque escasos se identificaron granos de almidón en el rizoma y en la raíz (Fig. 4D).

CLAVE PARA LA DETERMINACIÓN DE LAS ESPECIES BASADA EN LOS CARACTERES ESTRUCTURALES DE LA RAÍZ Y RIZOMA

1 Presencia de estructuras secretoras de aceites esenciales .......................... .................... Eryngium pandanifolium

1'Ausencia de estructuras secretoras de aceites esenciales ..................... 2

2 Raíz con drusas y con crecimiento secundario anómalo.

Alternanthera philoxeroides
2' Raíz sin drusas y con crecimiento secundario normal ........................... 3

3 Raíz con rafidios en la corteza y el floema ....................... Ludwigia peploides

3' Raíz y rizoma sin drusas ni rafidios. Presencia de inulina .... Senecio bonariensis

\section{DISCUSIÓN}

La estructura anómala de los tallos es un capítulo especial para diversas familias como las Amaranthaceae, Chenopodiaceae, Bignoniaceae, Convolvulaceae, Sapindaceae, etc. (Metcalfe y Chalk, 1965; Metcalfe, 1989). Alternanthera philoxeroides (Amarantáceas) la hemos tratado en trabajos previos (Colares 
et al., 2014), donde observamos la existencia de la estructura secundaria anómala y la caracterización de la familia por la presencia de drusas, en coincidencia con Metcalfe y Chalk (1950). En los órganos subterráneos de Eryngium pandanifolium se encontraron grandes cámaras aerenquimáticas. Esta característica fue hallada también en $E$. paniculatum Cav. \& Dombey ex F. Delaroche, por Morales y Ladio (2012), lo que está en relación a su hábitat. Así también se observaron los conductos secretores de aceites esenciales a los cuales se asocian las propiedades de sabor y aroma de E. paniculatum en la Patagonia argentina, donde es usado como medicinal (Morales y Ladio, 2012). Los órganos subterráneos de Senecio bonariensis también presentan aerénquima, correspondiéndose con el ambiente palustre. En el parénquima se identificaron granos de almidón y cristales de inulina. La presencia de inulina en el rizoma es coincidente con Ragonese (1988) y Strasburger et al. (1994), quienes señalaron que este compuesto se acumula preferentemente en los órganos subterráneos de las Asteraceae.

\section{CONCLUSIÓN}

En este trabajo se ha contribuido con descripciones morfológicas características estructurales e histoquímicas de los órganos subterráneos de las especies estudiadas y con las mismas se ha generado una clave de identificación, lo que constituye una herramienta de valor en el control de calidad botánica.

\section{AGRADECIMIENTOS}

Agradecemos a Silvana Auguet por su contribución con algunos preparados. Agradecemos a la Secretaría de Ciencia y Técnica de la Universidad Nacional de La Plata por el financiamiento económico de los estudios en el marco del Programa de incentivos a los docentes-investigadores.

\section{BIBLIOGRAFÍA}

Alonso J., Desmarchelier C. 2005. Plantas medicinales autóctonas de la Argentina.
L. O. L. A. (Literature of Latin America), Buenos Aires, 663 pp.

Arambarri A. M., Novoa M. C., Hernández M. P., Colares M. N., Perrotta V. G. 2013. Anatomía de Dicotiledóneas: tallos de hierbas terrestres medicinales rioplatenses (Buenos Aires, Argentina). Bonplandia 22: 5-28.

Arambarri A. M., Hernández M. P., Perrotta V. G., Colares M. N., Novoa M. C. 2016. Anatomía de los órganos subterráneos de Monocotiledóneas medicinales rioplatenses (Argentina). I. Acuáticas y palustres. Lilloa 53: 157-172.

Barboza G. E., Cantero J. J., Núñez C. O., Ariza Espinar L. 2006. Flora medicinal de la provincia de Córdoba (Argentina), Museo Botánico de Córdoba, Gráficamente ediciones, Córdoba, 1264 pp.

Barboza G. E., Cantero J. J., Núñez C., Pacciaroni A., Ariza Espinar L. 2009. Medicinal plants: A general review and a phytochemical and ethnopharmacological screening of the native Argentine Flora. Kurtziana 34: 7-365.

Colares M. N., Hernández M. P., Novoa M. C., Perrotta V. G., Auguet S., Arambarri A. M. 2014. Anatomía comparada de raíces medicinales de hierbas terrestres rioplatenses (Buenos Aires, República Argentina). Dominguezia 30: 5-18.

Cosa M. T., Dottori N., Stiefkens L., Hadid M., Matesevach M., Delbón N., Wiemer P., Machado S., Cabrera V., Costa C., Pérez A., Trenchi A. 2014. Aplicaciones de técnicas de histología vegetal a la resolución de diversos problemas. Laboratorio de Morfología Vegetal, Facultad de Ciencias Exactas, Físicas y Naturales, Universidad Nacional de Córdoba, $133 \mathrm{pp}$.

D’Ambrogio de Argüeso A. 1986. Manual de técnicas en histología vegetal. Ed. Hemisferio Sur, Buenos Aires, 86 pp.

Domínguez J. A. 1928. Contribuciones a la materia médica argentina. Instituto de Botánica y Farmacología (Facultad de Ciencias Médicas de Buenos Aires], Ed. Peuser, Buenos Aires, 433 pp.

González A. M. 2002. Anatomía del vástago en especies selectas de plantas hidrófilas. En: Arbo M. M. Tressens S. G. (editores), Flora del Iberá. EUDENNE. Corrientes, Argentina. Capítulo 9: 431450.

Gurr E. 1971. Synthetic dyes in biology, medicine and chemistry. Academic Press, London, England. http://stainsfile. info/StainFile/jindex.html [consulta: junio 2016]. 
Hernández M. P., Arambarri A. M. 2011. Recursos fitoterapéuticos y comportamiento poblacional en dos sectores de la ribera rioplatense de Berisso, Buenos Aires, Argentina. Bonplandia 20: 137148.

Hernández M. P., Colares M. N., Civitella S. M. 2009. Plantas utilizadas en medicina popular en un sector del Partido de Berisso, Buenos Aires, Argentina. Boletín Latinoamericano y del Caribe de Plantas Medicinales y Aromáticas 8: 435-444.

Hernández M. P., Civitella S. M., Rosato V. G. 2010. Uso medicinal popular de plantas y líquenes de la Isla Paulino, provincia de Buenos Aires, Argentina. Boletín Latinoamericano y del Caribe de Plantas Medicinales y Aromáticas 9: 258-269.

Hernández M. P., Novoa M. C., Arambarri A. M., Oviedo M. A. 2015a. Plantas medicinales y para condimento usadas en el sudeste del partido de Berisso (Buenos Aires, Argentina]. Bonplandia 24: 125138.

Hernández M. P., Novoa M. C., Civitella S. M., Mason D., Oviedo A. 2013. Plantas usadas en la medicina popular en la Isla Santiago, Buenos Aires, Argentina. Boletín Latinoamericano y del Caribe de Plantas Medicinales y Aromáticas 12 : 385-399.

Hernández M. P., Novoa M. C., Colares M. N., Perrotta V. G., Nughes L. M., Arambarri A. M. 2015b. Anatomía foliar de hierbas terrestres medicinales que crecen en la región rioplatense (Buenos Aires, Argentina). Bonplandia 24: 97-123.

Hieronymus G. 1882. Plantas diaphoricae florae argentinae. Boletín de la Academia de Ciencias de Córdoba 4: 199-598.

Hurrell J. A., Bazzano D. H., Delucchi G. 2006. Dicotiledóneas herbáceas vol. 1 (Biota rioplatense 11): nativas y exóticas. L.O.L.A. (Literature of Latin America), Buenos Aires, 288 pp.

Instituto de Botánica Darwinion - IBODA CONICET. www.darwin.edu.ar / [consultado: junio 2017]

Johansen D. A. 1940. Plant microtechnique. McGraw-Hill Book Company, New York.

Lahitte H. B., Hurrell J. A. 1996. Las plantas de la medicina popular de la isla Martín García. Comisión de Investigaciones Científicas de la provincia de Buenos Aires (CICPBA), $246 \mathrm{pp}$.

Lahitte H. B., Hurrell J. A., Belgrano M. J., Jankowski L. S., Haloua M. P., Mehltreter K. 2004. Plantas medicinales rioplatenses. L.O.L.A. (Literature of Latin America), Buenos Aires, 240 pp.
Luque R., Sousa H. C., Kraus J. E. 1996. Métodos de coloração de Roeser (1972) -modificado- e Kropp (1972) visando a substituição do azul de astraporazul de alcião 8 GS ou 8GX/Staining methods of modified Roeser (1972) and Kropp (1972), aiming at substituting the astra blue by alcian blue 865 or $8 G X$. Acta Botanica Brasilica 10: 199-212.

Martínez Crovetto R. 1981. Plantas utilizadas en medicina en el noroeste de Corrientes (República Argentina). Miscelánea (Fundación Miguel Lillo) 69: 1-139.

Metcalfe C. R. 1989. Anomalous structure In: Metcalfe C. R., Chalk L. Anatomy of the Dycotiledons. Vol. II: 52-63. Oxford University Press.

Metcalfe C. R., Chalk L. 1950. Anatomy of the Dycotiledons 1: 1-763. Oxford University Press.

Metcalfe C. R., Chalk L. 1965. Anatomy of the Dycotiledons 2: 764-1499. Oxford University Press.

Metcalfe C. R., Chalk L. 1979. Anatomy of the Dicotyledons. Clarendon Press, Oxford, Vol. 1: $276 \mathrm{pp}$.

Morales S., Ladio A. H. 2012. Plantas aromáticas con órganos subterráneos de importancia cultural en la Patagonia Argentina: una aproximación a sus usos dese la etnobotánica, la percepción sensorial y la anatomía. Darwiniana 50: 7-24.

Novoa M. C., Arambarri A. M. 2016. Importance of anatomical leaf-blade features for characterization of medicinal Commelinaceae in the Rio de la Plata area [Buenos Aires, Argentina). Boletín de la Sociedad Argentina de Botánica 51: 419-427.

Novoa M. C., Colares M. N., Arambarri A. M. 2012. Anatomy of Monocotyledons: stems and rhizomes of land herbs used as medicinal in the Rio de la Plata area (Argentina). Bonplandia 21: 53-61.

Passarelli L. M., Rolleri C. H., Ciciarelli M. M., Dedomenici A. C., González G. 2014. Flora vascular de humedales permanentes y transitorios bonaerenses (Buenos Aires, Argentina). Botanica Complutensis 38: 139-154.

Pochettino M. L., Martínez M. R., Itten B., Zucaro M. 1997. Las plantas medicinales como recurso terapéutico en una población urbana: estudio etnobotánico en Hernández (Pdo. La Plata, Prov. Buenos Aires, Argentina]. Parodiana 10: 141-152.

Ragonese A. M. 1988. Canales secretores en los órganos vegetativos de Eupato- 
rium inulaefolium H.B.K. (Compositae). Acta Farmacéutica Bonaerense 7: 161168.

Rondina R. V. D., Bandoni A. L., Coussio J. D. 2008. Especies medicinales argentinas con potencial actividad analgésica. Dominguezia 24: 47-69.

Rondina R. V. D., Bandoni A. L., Coussio J. D. 2010. Argentine medicinal plants with potential antifungal activity. Dominguezia 26: 31-39.

Ruzin S. E. 1999. Plant microtechnique and microscopy. University Press, Oxford, $323 \mathrm{pp}$.

Schussler E. E., Longstreth D. J. 2000. Changes in cell structure during the formation of root aerenchyma in Sagittaria lancifolia (Alismataceae). American Journal of Botany 87: 12-19.

Seago Jr. J. L., Marsh L. C., Stevens K. J., Soukup A. Votrubová D., Enstone D. E. 2005. A re-examination of the root cortex in wetland flowering plants with respect to aerenchyma. Annals of Botany 96: $565-579$.

Sérsic A. N., Cocucci A. A., Acosta M. C., Baranzelli M., Benítez Vieyra S., Boero L., Córdoba S., Cosacov A., Díaz L., Ferreiro G., Glinos E., Ibañez A. C., Marino S., Maubecin C. C., Medina M., Moré M., Paiaro V., Renny M., Sazatornil F., Sosa Pivatto M., Soteras F., Strelin M., Trujillo C., Wiemer A. P. 2015. Flores del centro de Argentina II. Nueva guía ilustrada para conocer 229 especies. Vol 1: 84-85. Vol. 2: 476-477. Instituto Multidisciplinario de Biología Vegetal (IMBIV), Córdoba, 664 pp.

Strasburger E., Noll F., Schenck H., Schimper A. F. W. 1994. Tratado de Botánica, Omega, Barcelona, España, 1068 pp.

Toursarkissian M. 1980. Plantas medicinales de la Argentina. Ed. Hemisferio Sur, Buenos Aires. 178 pp.

Tropicos - Home. www.tropicos.org/ [consultado: junio 2017]

Zarlavsky G. E. 2014. Histología vegetal. Técnicas simples y complejas. Sociedad Argentina de Botánica, 198 pp.

\section{APÉNDICE 1}

Lista en orden alfabético de nombres científicos de las especies estudiadas con su familia, nombre vulgar de uso frecuente, uso popular y actividad biológica. Los usos están referidos a los órganos subterráneos.

Alternanthera philoxeroides (Amaranthaceae) «lagunilla». Usos: diurético, depurativo, digestivo y hepático (Barboza et al., 2009; Sérsic et al. 2015) y para combatir enfermedades renales y de las vías urinarias (Lahitte y Hurrell, 2004; Hurrell et al., 2006). Contiene oxidasas en toda la planta (Domínguez, 1928) y la raíz posee flavonoides (Lahitte y Hurrell, 1996; Lahitte et al., 2004).

Eryngium pandanifolium (Apiaceae) «caraguatá». Usos: la raíz es aperitivo, diurético, astringente, depurativo, emenagogo, digestivo (Martínez Crovetto, 1981; Barboza et al., 2009). Contiene peroxidasas en la raíz (Dominguez, 1928).

Ludwigia peploides (Onagraceae) «falsa verdolaga». Usos: en uso externo, la planta entera en cataplasmas o lavajes se usa para tratar dermatosis, y como desinfectante de heridas y lastimaduras (Hurrell et al., 2006; Sérsic et al., 2015). Contiene polifenoles, taninos y oxalato de calcio (Lahitte et al., 2004). La planta entera tiene propiedad citotóxica (Barboza et al., 2009).

Senecio bonariensis (Asteraceae) «margarita de bañado». Abluente y cicatrizante (Barboza et al., 2006, 2009; Hurrell et al., 2006). Posee actividad antibacteriana (Barboza et al., 2006; 2009). 